\title{
Mobile application-guided open oral food challenge used in infants with non-IgE mediated cow's milk protein allergy during COVID-19 pandemic
}

\author{
Li-Jing Xiong ${ }^{1}$, Xiao-Zhi Deng ${ }^{1}$, Yang Li $^{1}$, Jing $\mathrm{Li}^{1}$, Xiao-Qing $\mathrm{He}^{1}$, Chu Wang ${ }^{1}$, Meng-Yao \\ Zhou $^{1}$, and Xiao-Li Xie ${ }^{1}$ \\ ${ }^{1}$ Chengdu Women's and Children's Central Hospital
}

September 8, 2020

\begin{abstract}
Background: Open oral food challenge (OFC) is a useful tool in management of non-IgE mediated cow's milk allergy (CMPA) infants. Because of COVID-19 pandemic, clinical services for noninfectious diseases were hard to maintain as usual. Internet hospital model of medicine got more profoundly involved in the medical service systems. Methods: we used a hospital-led mobile application (APP) platform to guide the open OFC conducted at home, and assessed the effect of this novel model by comparing with the traditional pattern of open OFCs. Results:Sixty two infants with aged 6.552.81 months were included. Result of Kappa coefficient was $0.596(\mathrm{P}=0.000)$ suggesting a good consistency of judgments between parents and doctors in APP-guided group. The accuracy of parents was $85.48 \%$ and $74.36 \%$ in APP-guided group and control group, respectively. If parents reported positive results, the consistency with outcomes confirmed by specialists was $87.76 \%$ and $74.51 \%$, while If parents reported no symptoms, the rate of negative outcomes identified by specialist was $76.92 \%$ and $74.07 \%$ in two groups. With the utilization of mobile application, the false-positive rate $(12.25 \%)$ was reduced by half with statistical significance $(\mathrm{X} 2=39.32, \mathrm{P}=0.000)$. The proportion of false-positive caused by vomiting decreased from $61.5 \%(8 / 13)$ to $33.33 \%(2 / 6)$. Conclusion: This was a preliminary attempt to use hospital-led telemedicine tool for guiding open OFCs in CMPA children. Although there are still many problems needing discussion, it could be a promising approach used in pediatric population with allergic diseases during the special period like COVID-19 pandemic.
\end{abstract}

\section{Hosted file}

main text20200907.doc available at https://authorea.com/users/357305/articles/479900-mobileapplication-guided-open-oral-food-challenge-used-in-infants-with-non-ige-mediated-cow-smilk-protein-allergy-during-covid-19-pandemic

\section{Hosted file}

Table.doc available at https://authorea.com/users/357305/articles/479900-mobile-applicationguided-open-oral-food-challenge-used-in-infants-with-non-ige-mediated-cow-s-milkprotein-allergy-during-covid-19-pandemic 